\title{
Erektile Dysfunktion als KHK-Prädiktor
}

\author{
Eine Studie mit Infarktpatienten zeigt unmissverständlich, dass man bei \\ einer erektilen Dysfunktion auch an das koronare Risiko denken sollte.
}

Zwischen erektiler Dysfunktion (ED) und koronarer Herzkrankheit (KHK) besteht wahrscheinlich über die endotheliale Dysfunktion eine pathophysiologische Verbindung. Wie eng die beiden Probleme korrelieren, zeigte sich nun in einer Studie an 139 männlichen Patienten im Alter von $60 \pm 12$ Jahren, die sich 13 Tage nach einem akuten Myokardinfarkt in einer Klinik für kardiale Rehabilitation befanden. Sie alle machten in einem Fragebogen Angaben zur erektilen Funktion und unterzogen sich einfachen Leistungstests.

57\% der Patienten litten an einer ED. Diese Gruppe erwies sich als deutlich weniger leistungsfähig. Sie erreichte im 6-Minuten-Gehtest nur einen Schnitt von $490 \pm 119 \mathrm{~m}$. Die Patienten ohne ED schafften $564 \pm 94 \mathrm{~m}$. In einem symptombegrenzten kardiopulmonalen Belastungstest lag die ED-Gruppe mit $79 \pm$ 28 vs. $109 \pm 34 \mathrm{~W}$ zurück. Auch die Sauerstoffaufnahme während der Belastungsspitze lag signifikant niedriger.
- Compostella L et al. History of erectile dysfunction as a predictor of poor physical performance after an acute myocardial infarction. Eur J Prevent Cardiol 2017;24:460-7

\section{KOMMENTAR}

Die relativ schlicht angelegte Untersuchung bestätigt die bekannte Tatsache, dass die ED offensichtlich mit der KHK korreliert ist oder zumindest als Risikofaktor gewertet werden kann. Zusätzlich erwies sie sich in der vorliegenden Untersuchung als Prädiktor für eine verminderte kardiale Leistungsfähigkeit.

Obwohl das keine weltbewegende Neuigkeit ist, hat die Untersuchung doch Konsequenzen für die Praxis. Patienten mit ED suchen wegen des Leidensdrucks häufig einen Arzt auf, wobei es sich aber meistens um den Urologen handelt. Wegen der grundsätzlich anderen Interessenlage wird so bestimmt häufig eine wichtige Gelegenheit verpasst, den Patienten auf die Möglichkeit eines koronaren Risikos und einschlägige Untersuchungen hinzuweisen. Urologen sollten hier ein wenig über den Tellerrand schauen.

Umgekehrt könnte aber auch der Hausarzt oder der Internist über den Umweg der Frage nach der erektilen Funktion Hinweise auf eine mögliche KHK bekommen. Schließlich bedarf es für die Entdeckung von Risiken und die Weichenstellung der Diagnostik oft nur einer einzigen Frage.

Prof. Dr. med. H. S. FüeßI

\section{Hier steht eine Anzeige.}

\author{
Springer
}

